\title{
Comparación de la calidad de la obturación radicular obtenida con el sistema fluido de obturación radicular v/s técnica de compactación lateral
}

\section{Comparison of the sealed quality of the root canals filled with a Flow System Technique versus lateral compactation technique}

\author{
Hernández Vigueras S*, Piñeiro Miranda S**, Aracena Rojas S***, \\ Alcántara Dufeu $\mathrm{R} * * * *$
}

\section{RESUMEN}

El propósito de este estudio es comparar la calidad de la obturación radicular obtenida al obturar con GuttaFlow ${ }^{\circledR}$ (GF) y con la Técnica de Compactación Lateral (LC).

Metodología: Se utilizaron 44 raíces palatinas de molares superiores extraídos las cuales fueron preparadas y divididas aleatoriamente en 2 grupos para ser obturadas con la técnica del sistema fluido (GF) y la Técnica de Compactación Lateral en frío. Las muestras fueron radiografiadas y cortadas transversalmente para su evaluación microscópica por tercios radiculares. Los parámetros de calidad de obturación evaluados fueron la extensión de la obturación, la adaptación a las paredes del conducto radicular y la presencia de poros/fisuras/ vacuolas en el interior de la masa obturadora.

Resultados: No se encontraron diferencias significativas en la calidad de la obturación de los 2 grupos estudiados, exceptuando la adaptación a las paredes en tercio cervical y medio, y la presencia de poros en tercio cervical, en los cuales el grupo LC presentó mejores resultados.

Conclusiones: El nuevo material fluido de obturación no demostró mejorar la calidad de la obturación radicular al ser comparado con la técnica de LC.

Palabras clave: Obturación radicular, GuttaFlow, sistemas de obturación, compactación lateral.

\section{SUMMARY}

The aid of this study is compare the sealed quality of root canals filled with two obturations systems, GuttaFlow or Flow System Technique (GF) and Lateral Compactation Technique (LC).

Methodology: Forty-four palatal roots of molar teeth were prepared and divided randomly in two groups to be obturated with the flow system technique and Lateral Compactation Technique. The samples were radiographed and cut cross-sectionally by roots thirds for its microscopic evaluation. The obturations quality parameters evaluated were extension obturation, adaptation to the walls of the root canal and presence of pores/fissures/ vacuolas inside the obturation.

* Docente Endodoncia. Escuela de Odontología. Facultad de Medicina. Universidad Austral de Chile. Valdivia Chile.

** Especialista en Endodoncia. Hospital Castro. Chiloé. Chile.

*** Docente Endodoncia. Depto. Odontología Integral. Facultad de Medicina. Universidad de La Frontera Temuco. Chile.

**** Docente Facultad de Odontología. Universidad de Concepción. Concepción. Chile. 
Results: No statistical difference amongst the filling techniques except between adaptation to the walls of the root canal in medium and cervical thirds and absence of pores/fissures/vacuolas in cervical third in which LC displayed better results.

Conclusions: The new flow material obturation it did not demostrated to improve the quality of root obturation to the being compared with the LC technique.

Key words: Root obturation, GuttaFlow, obturation technique, lateral compactation technique.

Fecha de recepción: Diciembre 2007.

Aceptado para publicación: Diciembre 2007.

Hernández Vigueras S, Piñeiro Miranda S, Aracena Rojas S, Alcántara Dufeu R. Comparación de la calidad de la obturación radicular obtenida con el sistema fluido de obturación radicular v/s técnica de compactación lateral. Av. Odontoestomatol. 2008; 24 (4): 255-260.

\section{INTRODUCCIÓN}

Con los actuales avances de la ciencia y la tecnología, han surgido numerosas herramientas que pretenden mejorar las diferentes etapas de la terapia de conductos radiculares. Junto con ello se han desarrollado nuevos materiales y técnicas para mejorar la etapa de obturación radicular (1). De esto, surge la necesidad de realizar diversos estudios que comprueben la efectividad clínica de las nuevas tecnologías que el mercado odontológico día a día nos ofrece.

A pesar de la unanimidad de opiniones sobre la importancia de la obturación, existen divergencias sobre el material a utilizar en la obturación del conducto conformado, lo que denota el reconocimiento de la inexistencia de un material ideal en la actualidad (2).

El propósito de este estudio fue analizar comparativamente la calidad de la obturación radicular obtenida al utilizar dos sistemas de obturación radicular, GuttaFlow $^{\circledR}$, un sistema fluido de obturación en frío y la compactación lateral en frío.

\section{MATERIAL Y MÉTODO}

El material utilizado en la investigación fue GuttaFlow $^{\circledR}$ (Roeko Coltène/Whaledent, Langenau, Alemania), material relativamente nuevo en el mercado y poco conocido como material de obturación, el cual según su fabricante, tiene propiedades que brinda- rían obturaciones homogéneas y más fáciles de realizar que las obtenidas con la tradicional técnica de Compactación Lateral en frío. Es un sistema autopolimerizable de obturación de conductos que combina dos productos, partículas de gutapercha de tamaño inferior a $30 \mu \mathrm{m}$, y sellador en base de polidimetilsiloxano. Según el fabricante, produce un mejor sellado, dada su alta fluidez, no necesita de calor, y no sufre contracción, por el contrario, el material se expande levemente durante el fraguado (3).

Este material es considerado un cemento, por lo que el riesgo de sobreobturaciones es alto (4). Es tixotrópico, la viscosidad disminuye cuando el material es sometido a presión, por eso el GF se deslizaría hasta en los más pequeños conductos laterales. Otra de sus propiedades es la biocompatibilidad, siendo más biocompatible que otros materiales tradicionales de obturación. Además, en cuanto a la solubilidad del material, esta sería prácticamente nula. Esto permite una obturación del canal dimensionalmente estable y no porosa (3).

Se utilizaron 48 raíces palatinas de primeros y segundos molares superiores humanos extraídos con ápice maduro. Se obtuvieron radiografías estandarizadas de las 48 raíces, con las cuales se determinó el grado de curvatura que presentaban, estableciendo dentro de los criterios de inclusión, un grado de curvatura radicular Tipo I o II, Leve y Moderado, según la Clasificación de Schneider (5). Posteriormente, se realizó la preparación de los conductos radiculares, 
estableciendo la longitud de trabajo a $1 \mathrm{~mm}$ del ápice anatómico, con una lima K File \#10 (Dentsply, Maillefer). La instrumentación se llevó a cabo utilizando un Motor X Smart (Dentsply, Maillefer), con limas ProTaper Universal (Dentsply, Maillefer), preparando hasta lima F3 en apical, siguiendo la técnica que propone el fabricante. Cada conducto fue irrigado con 2 cc de hipoclorito de sodio al 2,5\%, entre cada cambio de limas, con jeringas desechables y agujas de calibre 25 GX.

Al terminar la preparación, los conductos fueron secados con puntas de papel, y las muestras fueron divididas al azar en 2 grupos. El grupo 1 de GF con 24 muestras y el grupo 2, de LC, con 20 muestras.

Las piezas del grupo 1 fueron obturadas siguiendo las instrucciones del fabricante, que indican que después de seleccionar el cono maestro a longitud de trabajo, se debe mezclar el producto contenido en la cápsula en un amalgamador mecánico durante 30 segundos.

Una vez mezclado el material se inyectó en el interior del conducto con el dispensador proporcionado por el fabricante hasta que el material podía verse a la entrada del conducto. Posteriormente se introdujo el cono maestro seleccionado previamente hasta la longitud de trabajo. Cuando fue necesario se volvió a rellenar con el material fluido hasta completar la obturación de todo el conducto.

El segundo grupo fue obturado con la técnica de LC en frío, utilizando un cono maestro Protaper F3 seleccionado de acuerdo al calibre del conducto conformado y puntas accesorias de gutapercha. El cemento sellador empleado correspondió al cemento Tubliseal (Kerr).

Después de realizadas las obturaciones radiculares, se sellaron los accesos con cemento provisional Fermin ${ }^{\circledR}$ (Detax, Alemania). Posteriormente, las muestras se almacenaron en un horno de cultivo a $37^{\circ}$ Celsius por 24 horas, con el fin de permitir el tiempo adecuado de fraguado del cemento sellador.

Se realizó luego el corte transversal de la raíz con disco de diamante de dos caras activas (Sorensen) y abundante irrigación, dividiéndola topográficamente en 3 tercios: cervical, medio y apical. Los cortes se realizaron dividiendo la longitud de cada raíz en 3 partes iguales, que representan cada tercio radicular, y luego en la mitad de cada uno de estos tercios se realizó el corte a observar. El número total de muestras obtenidas fue de 72 para el grupo 1 y de 60 para el grupo 2.

Las muestras fueron observadas al microscopio clínico (Opto, Brasil), con aumento 30×. La determinación de la longitud de la obturación radicular se realizó mediante la observación de radiografías estandarizadas de cada muestra una vez obturadas.

Los parámetros evaluados en ambos grupos fueron:

1. Adaptación de la masa obturadora a las paredes del conducto radicular por cada tercio radicular, de acuerdo a la siguiente categorización:

- Adaptación completa a las paredes del conducto radicular.

- Desadaptación de la masa obturadora a las paredes del conducto radicular.

2. Presencia de poros, fisuras o vacuolas al interior de la masa obturadora en cada tercio radicular, según la siguiente categorización:

- Sin presencia de poros, fisuras o vacuolas.

- Presencia de poros, fisuras o vacuolas.

3. Longitud de la obturación radicular, según la siguiente categorización:

- Obturación completa se determinó cuando la masa obturadora llega al límite apical de la preparación radicular.

- Subobturación se determinó cuando la masa obturadora no alcanza a llegar al límite apical de la preparación radicular.

- Sobreobturación se determinó cuando la masa obturadora traspasa el límite apical de la preparación radicular.

El registro de los datos recopilados durante el análisis de las muestras fue realizado con el programa Microsoft Excel y el procesador de texto Microsoft Word, en tanto, el análisis estadístico de los datos fue realizado con el programa SPSS versión 12, aplicando el test chi cuadrado y el test exacto de Fisher. 


\section{RESULTADOS}

1. Adaptación a paredes radiculares por cada grupo y según tercios radiculares.

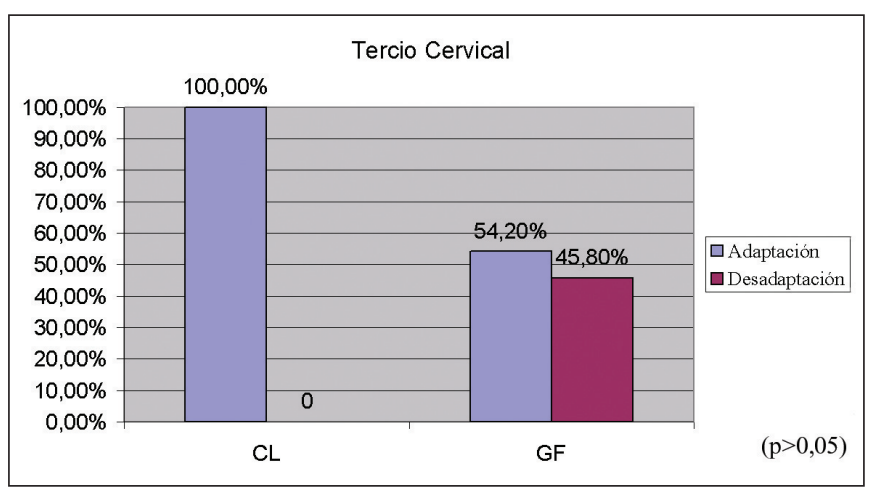

Fig. 1.

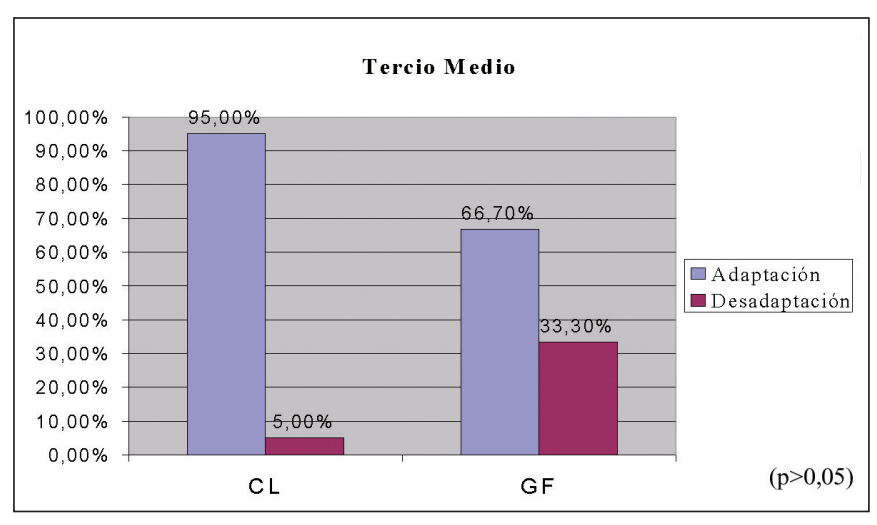

Fig. 2.

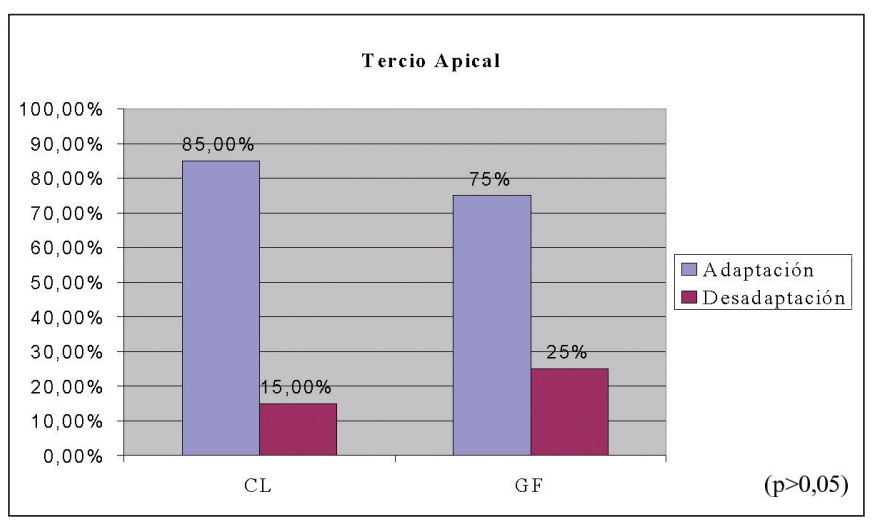

Fig. 3.
2. Presencia de poros/fisuras/vacuolas por cada grupo y según tercios radiculares.

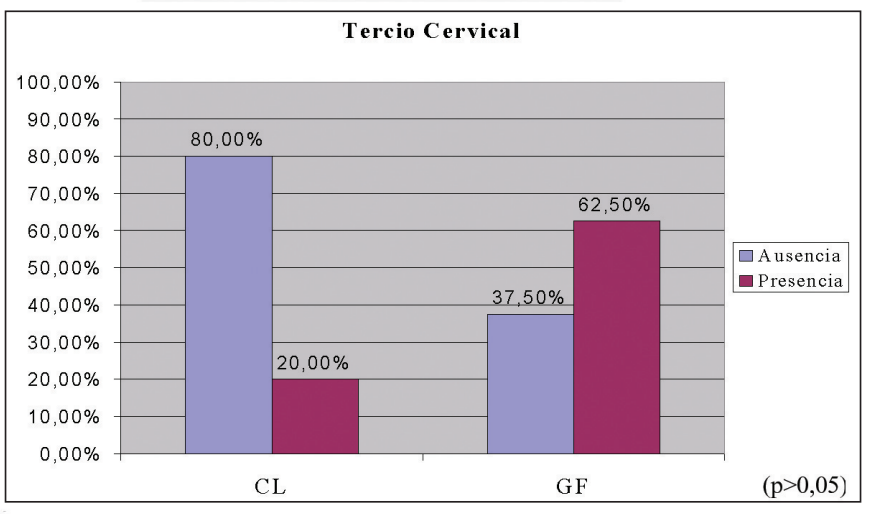

Fig. 4.

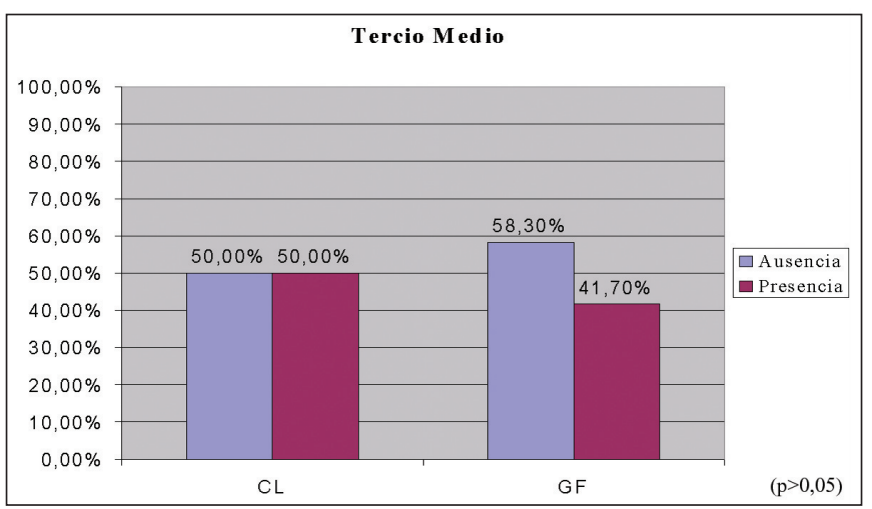

Fig. 5.

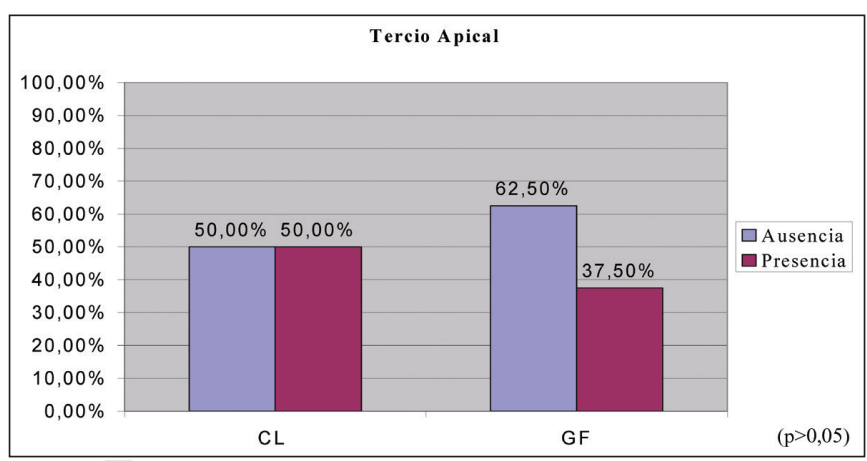

Fig. 6.

3. Longitud de obturación radicular en cada grupo: Análisis radiográfico. 


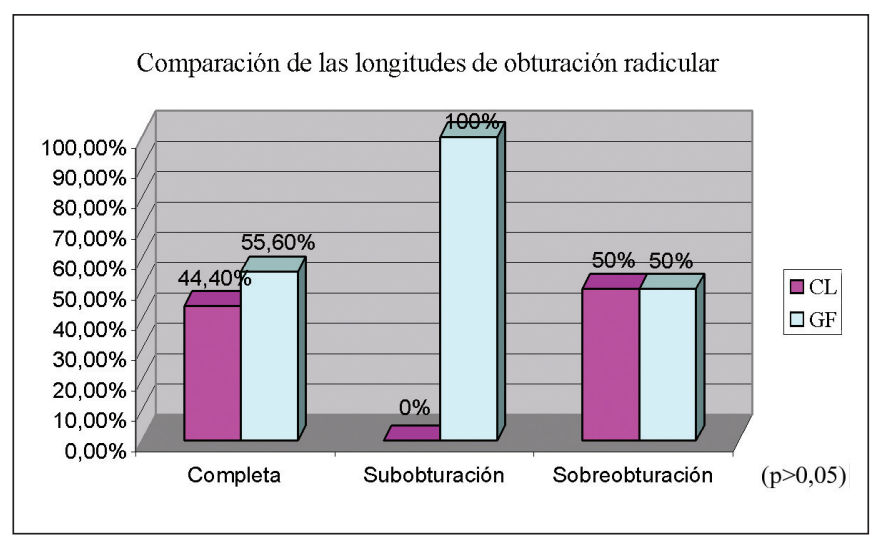

Fig. 7.

\section{DISCUSIÓN}

A pesar que los resultados indican que no hubo diferencia estadísticamente significativa en cuanto a la longitud de la obturación, en la etapa experimental se observó un mayor volumen de extrusión de material GuttaFlow, que lo observado con Compactación Lateral. Esto no pudo ser reflejado en los resultados, dado que el volumen de extrusión no se cuantificó en las muestras.

La mayor cantidad de poros o vacuolas al interior de la masa obturadora en el tercio cervical del grupo obturado con GuttaFlow, coincide con los resultados obtenidos en un estudio similar realizado por El Ayouti (5), donde además, se señala la necesidad de realizar estudios de filtración y sobreobturación que pudiese presentar este producto, lo que concuerda con nuestra experiencia.

Sería necesario realizar otros estudios a este material, en los cuales se simulen las condiciones clínicas de la boca, en cuanto a posición de los dientes en la arcada para evaluar si se logra un adecuado acceso a todas las piezas dentarias con la pistola dispensadora. Evaluar como actúa el GuttaFlow ${ }^{\circledast}$ en la presencia de tejidos periodontales o un material que actuase como barrera contra la extrusión, con el objetivo de evaluar adecuadamente este parámetro y extrapolar los resultados a la clínica. Sin embargo, otras características del GuttaFlow ya han sido estudiadas por otros autores; Eldeniz y cols. evaluaron los efectos citotóxicos del GF comparándolo con otros cementos selladores existentes en el mercado y algunos recientemente lanzados, demostrando que posee una citotoxicidad menor que otros cementos como Epiphany, EndoRez, Acroseal y Apexit (6). La filtración de este nuevo material fue evaluada en un estudio de Monticelli y cols. demostrando ser mayor en los tercios coronales y medios al ser comparado con la filtración en piezas obturadas con una técnica de compactación vertical caliente (7).

En nuestro estudio, GuttaFlow ${ }^{\circledR}$ no demostró mejorar la calidad de la obturación radicular al ser comparado con la Técnica de Compactación Lateral en frío. Sin embargo, esta técnica de GuttaFlow ${ }^{\circledR}$ es más fácil y rápida de realizar, pero requiere aparatología especial, lo que aumenta el costo de su aplicación.

\section{BIBLIOGRAFÍA}

1. Fernández K. Técnicas de Obturación Sistema de Conductos Radiculares. Monografía Programa Especialización en Endodoncia, Universidad de Concepción, 2004: 5-15.

2. Funes MG. Obturación del Sistema de Conductos Radiculares. Monografía Programa Perfeccionamiento en Endodoncia, Universidad de Concepción, 2005: 6-15.

3. Folleto informativo GuttaFlow ${ }^{\circledast}$, proporcionado por Coltène/Whaledent.

4. ElAyouti A, Achleithner C, Löst C, Weiger R. Homogeneity and Adaptation of a New GuttaPercha Paste to Root Canal Walls. J Endod 2005; 31: 687-90.

5. Schneider SW. A comparation of canal preparations in straight and curved root canals. Oral Surg 1971; 32: 271-5.

6. Eldeniz AU, Mustafa K, Ørstavik D, Dahl JE. Cytotoxicity of new resin-, calcium hydroxide-, and silicone-based root canal sealers on fibroblasts derived from human gingiva and L929 cell 
lines. International Endodontic Journal2007; 40: 329-37.

7. Monticelli F, Sword J, Martin RL, Schuster GS, WellerRN, Ferrari M, Pashley DH, Tay FR. Sealing properties of two contemporary single-cone obturation systems. International Endodontic Journal 2007; 40: 374-85.

\section{CORRESPONDENCIA}

Scarlette Hernández Vigueras.

Escuela de Odontología, Campus Isla Teja, Universidad Austral de Chile, Valdivia, Chile.

Fono/Fax: 5663293900 shv81@yahoo.com 